\title{
Focusing Characteristics of Symmetrically Configured Bootlace Lenses
}

\author{
J. PAUL SHELTON, FELLOW, IEEE
}

\begin{abstract}
A symmetrically configured class of bootlace lenses (also known as Gent or Rotman lenses) is analyzed. These trifocal lenses are used to feed half-wave spaced linear arrays to form a set of multiple beams. The lens dimensions are scaled' so that all lenses have transmission characteristics equivalent to those of a Butler matrix with the same number of ports. The relationship between the focusing characteristics and the lens dimensions is calculated, and it is shown that these symmetrically configured lenses form a one-parameter family. Design equations are derived, and a design procedure is described. It is found that, for a wavefront tolerance of $\pm \lambda / 16$, the maximum usable array length varies from $20 \lambda$ to $200 \lambda$ as the ratio of lens width to array length varies from one to 1.6 .
\end{abstract}

\section{INTRODUCTION}

$\mathbf{T}$ HE BOOTLACE lens was invented by $\mathrm{H}$. Gent in 1956 $[1],[2]$. As shown in Fig. 1, it uses a planat homogeneous wave-transmission region which has a set of feed ports on one side and a set of lens ports on the other. For the case in which the radiator array is constrained to a straight line, it has been shown that perfect focus can be obtained at three points on the feed curve [3].

Design equations of the bootlace lens were presented by Rotman and Turner in 1963, and they also gave examples of aberration characteristics [4]. Archer has recently described applications of the lens [5]. Whereas the primary intended application of the lens in the 50's was for rapid mechanical scan by means of feed motion, recent interest has been based on the lens' capabilities as a multiple-beam system. Gent lenses have been used recently in experimental precision aircraft landing systems in Australia and the United States [6] . ${ }^{1}$

Rotman and Turner required that the feed curve be circular and optimized the design parameters accordingly. This was consistent with rapid mechanical scan of feed horn. In terms of multiple-beam configurations, however, requiring the feed curve to be a circle is an unnecessary constraint. The design study described in this paper placed no limitation on the shape of the feed curve, and concluded that the lens should exhibit front-to-back symmetry. Furthermore, although examples of aberration characteristics were presented by Rotman and Turner, it is not possible to predict lens performance for all cases using their paper. That is, the design limitations of the lens were not established. Sufficient computation has been done to present in this paper a comprehensive description of the focusing characteristics of a symmetrically configured class of these lenses.

This paper does not treat the amplitude distributions produced at the aperture of the lens, nor does it address the farfield pattern characteristics, since these are determined by both

Manuscript received July 1, 1977; revised November 29, 1977. This work was supported by the Federal Aviation Administration.

The author is with the Target Characteristics Branch, Naval Research Laboratory, Washington, DC 20375.

1 Australia's Interscan Microwave Landing System employs a Gent lens for flare elevation guidance.

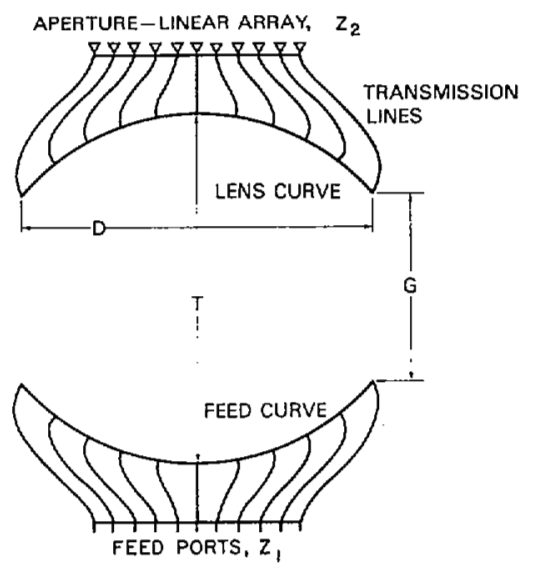

Fig. 1. Gent lens configuration.

the phase and amplitude distributions at the aperture. The analysis is based on geometrical optics ray-tracing techniques.

The next section of the paper reviews preliminary considerations with regard to performance and design parameters, and introduces a symmetry concept. The third section presents design characteristics. The design equations for these lenses are derived in the Appendix.

\section{PRELIMINARY CONSIDERATIONS}

\section{Performance Parameters}

A typical wavefront aberration curve produced by a bootlace lens is sketched in Fig. 2. The aberration curve is obtained by computing the difference between the actual nonlinear wavefront, as it leaves the array aperture, and an ideal linear wavefront. This path-length difference is normalized relative to the length of the aperture. Thus as used throughout this paper, wavefront error or aberration is a dimensionless parameter, indicated by the symbol $E$, and is an effective measure of the focusing performance of the lens. Note that wavefront error is defined as deviation from the desired wavefront, so that the total wavefront is contained in a region $\pm E$ relative to the desired linear wavefront. The lens is coma-limited in that the predominant aberration is described by a cubic function. If the maximum wavefront aberration is plotted versus the location on the feed curve, the result is shown in Fig. 3. Thus not only is the dominant aberration cubic, but the magnitude of aberration is also a third-order polynomial in the displacement along the feed or focal curve.

One objective of this section is to define a measure of aberration and a method for determining the portion of the feed curve that yields a given performance level for a particular lens design. A wavefront error surface has been used for this purpose, and a typical surface is depicted in Fig. 4. The level of the surface relative to the $z_{1}, z_{2}$ plane represents the amount by which the wavefront, located at aperture point $z_{2}$, deviates 


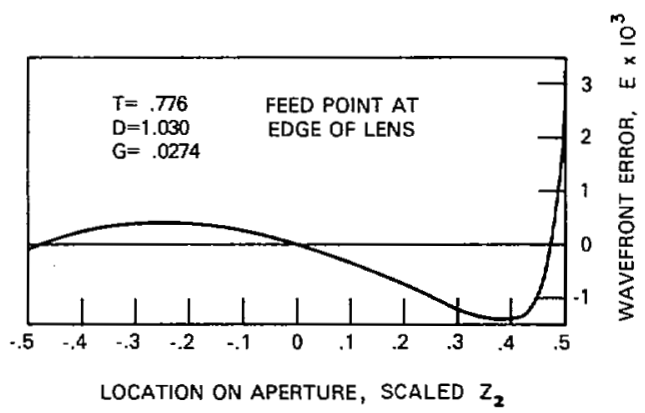

Fig. 2. Example of wavefront aberration curve.

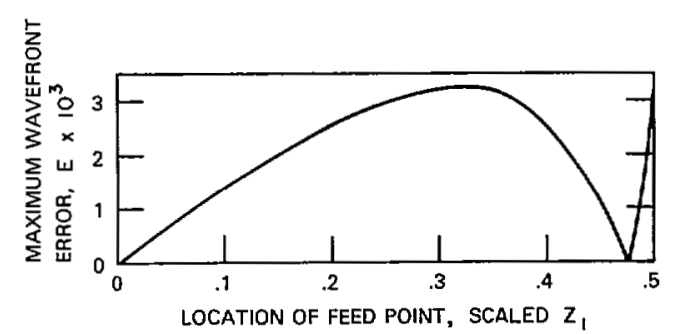

Fig. 3. Maximum wavefront error versus feed location.

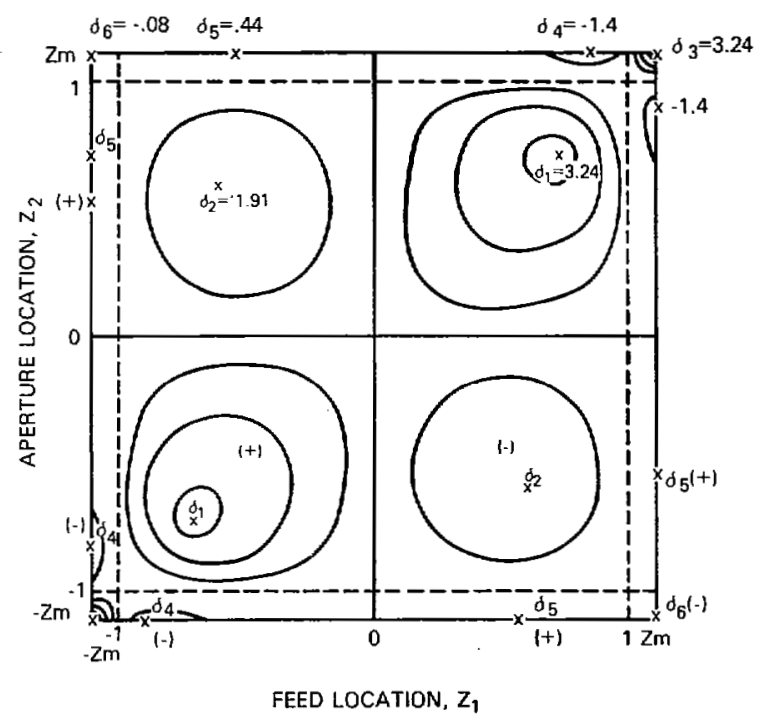

Fig. 4. Wavefront error surface.

from ideal linear collimation for any feed point located at $z_{1}$. The intersection of the plane $z_{1}=$ constant with the error surface gives the wavefront error curve for that feed point. Thus Fig. 4 contains a complete description of the focusing performance of the lens. The three foci for the example are at $z_{1}=0$ and $z_{1}= \pm 1$. The reference linear wavefronts from which the error surface is computed are chosen so that every error curve has zeros at $z_{2}=0$ and $z_{2}= \pm 1$. For lens configurations with right-left symmetry, the error surface is symmetric about the origin. If front-to-back symmetry is imposed, $z_{\mathbf{1}}$ and $z_{2}$ are interchangeable, and the error surface is also symmetric about the lines $z_{2}= \pm z_{1}$. The error surface has four relative extrema, one in each quadrant, and these are labeled $\delta_{1}$ and $\delta_{2}$. By symmetry, these extrema are located on the lines $z_{2}= \pm z_{1}$.

To determine the region over which the feed and lens curves are usable, the larger of $\left|\delta_{1}\right|$ or $\left|\delta_{2}\right|$ is selected and called $\delta_{m}$. Then the maximum values of $\left|z_{2}\right|$ and $\left|z_{1}\right|$, referred to as $z_{m}$, are increased until $\left|\delta_{3}\right|,\left|\delta_{4}\right|,\left|\delta_{5}\right|$, or $\left|\delta_{6}\right|=\delta_{m} . \delta_{3}$ and $\delta_{6}$ are the values of the error surface at the four points defined by $\left|z_{1}\right|=\left|z_{2}\right|=z_{m} . \delta_{4}$ and $\delta_{5}$ are the local extrema on the intersections of the error surface with the planes defined by $\left|z_{1}\right|=z_{m}$ and $\left|z_{2}\right|=z_{m}$. In general, $\delta_{3}=\delta_{4}=\delta_{5}=\delta_{6}=0$ for $z_{m}=1$, and the absolute values of $\delta_{3}, \delta_{4}, \delta_{5}, \delta_{6}$ increase monotonically with increasing $z_{m}$. It is found that $\delta_{m}$ is determined by $\delta_{1}$, and $z_{m}$ is determined by $\delta_{3}$ for lenses that are likely to be of practical interest.

Once the appropriate value of $z_{m}$ has been determined, it is possible to multiply all dimensions by an appropriate scale factor so that the aperture is contained in the region $|z| \leqslant 0.5$. This scaling process leads to the slight inconsistency of unscaled feed and aperture locations being used in Fig. 4, while the parameters shown in Figs. 2 and 3 are scaled.

\section{The Case for Symmetry}

The lens configurations presented in this paper have the symmetry illustrated in Fig. 5. The lens and feed curves are identical with opposite orientations. The port locations on the lens and feed curves are also identical. Further, a set of transmission lines is connected to the feed ports, identical with the lines connecting the lens ports with the array elements. The resulting configuration exhibits front-back as well as right-left symmetry, and the input ports and array ports can be interchanged. In the original concept of the bootlance lens, the transmission lines constitute the lens. In the configuration of Fig. 5, the lens is compound rather than simple in the optical sense, and it can be viewed as a symmetric doublet.

The reasons for choosing the symmetric configuration are collected and listed below. For the first four items listed below, the arguments cannot be rigorously proved. These conclusions are based on computer analysis of symmetric and asymmetric configurations, and in each case the symmetric lens exhibited a local extremum with respect to the lens characteristic under consideration. Items number 5 and 6 are evident by inspection of Fig. 5.

1) It was found that an asymmetric lens will have either the lens arc or the feed arc more strongly curved than the arcs obtained for the symmetric solution. In addition, the more strongly curved arc will have larger port spacings and a larger variation in port spacing from center to edge.

2) Symmetric lenses exhibit better aberration characteristics.

3) The self-illumination problem-the tendency for feed ports to illuminate other feed ports, for example-is more severe for asymmetric lenses.

4) The symmetric lens is smaller than an equivalent asymmetric lens.

5) It is possible to divide a symmetric lens on its line of symmetry with a reflecting plane and realize a reflection-type collimating system for which the feed ports and radiating ports are identical.

6) If some type of beam synthesis is to be carried out using combinations of beams, the symmetric configuration with two sets of transmission lines must be used to insure that all beams have the same far-field phase reference. The formation of monopulse patterns is an example of such synthesis.

\section{DESIGN DATA}

The derivation of these design equations for Gent lenses followed a not uncommon course for exploratory analysis-the initial objectives were not achieved by the final results. The initial objective was to derive a simplified design procedure 

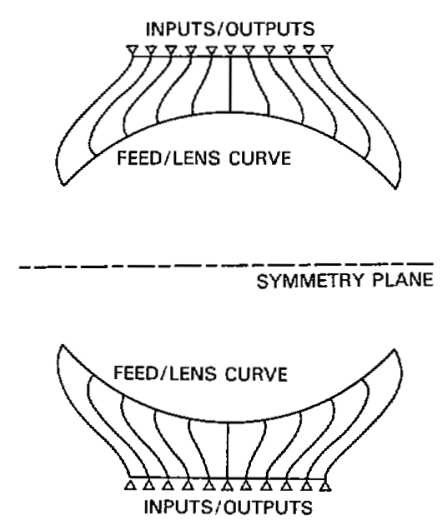

Fig. 5. Symmetric lens configuration.

without regard to symmetry. The purpose was to obtain general relationships about the lenses rather than detailed design information.

The initial analysis used the end points of the lens curve and the three focal points as inputs. From these, fourth power polynomials were derived for the feed and lens curves and for the transfer function from the lens curve to the radiating array. Approximate estimates of lens performance were made, and large numbers of cases could be tested very quickly because of the minimal computation involved.

It was noted that a judicious choice of initial conditions produced a lens with front-to-back symmetry as well as rightleft symmetry, at least to the six place accuracy of the computer printout. Then it was ascertained that symmetric configurations give better aberration characteristics than asymmetric. Finally, it was realized that the symmetric lens represents a one-parameter family and that performance evaluation on an exact basis could be carried out straightforwardly. At this point the approximate analysis was abandoned, and the exact solution for the symmetric lens, outlined in the Appendix, was derived.

The design equations derived in the Appendix have been programmed for computer calculation, and some of the results are plotted in Fig. 6. The basic parameters of interest-lens dimensions normalized to aperture length, element spacings relative to $\lambda / 2$, and maximum wavefront deviation relative to the aperture length-are presented in terms of lens thickness $T$. Fig. 7 is a plot of the lens shapes for $T=0.775,1.04$, and 1.65. It is not accidental that the endpoints of the lens curves shown in Fig. 7 appear to fall on a straight line. It is shown in the Appendix that $T-G \approx .75$, and it has been found that actual design data deviate by less than 0.002 . Thus the axial depth of lens and feed curves, which is $(T-G) / 2$, is very nearly $3 / 8$ the aperture length for all lenses.

As described in the Appendix, the symmetric Gent lens forms a one-parameter family, and this parameter can be any one of the variables plotted in Fig. 6. The dimensionless parameter $A$ is also plotted because the design equations use $A$ (defined in the Appendix) as one of the initial inputs. All lenses have been scaled so as to provide \pm 90 degrees coverage from a radiating array of half wavelength spaced elements. Fig. 6 is admittedly a complicated plot. It does show, however, that all lens parameters vary monotonically relative to one another, and it, together with Fig. 7, gives the reader a rough idea of lens performance versus size and shape. For a detailed design the reader must use the Appendix.

It is notable that the wavefront deviation is a rapidly varying function of lens size. Doubling the lens thickness reduces aber-

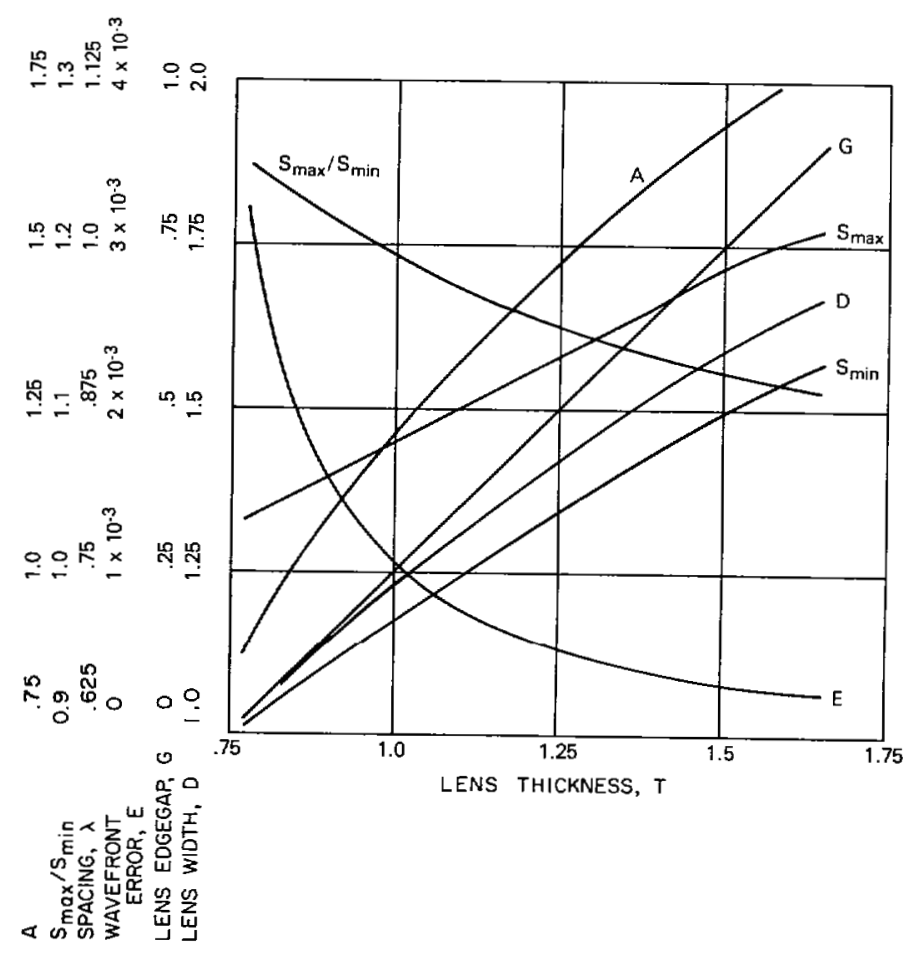

Fig. 6. Design data for symmetric Gent lenses.

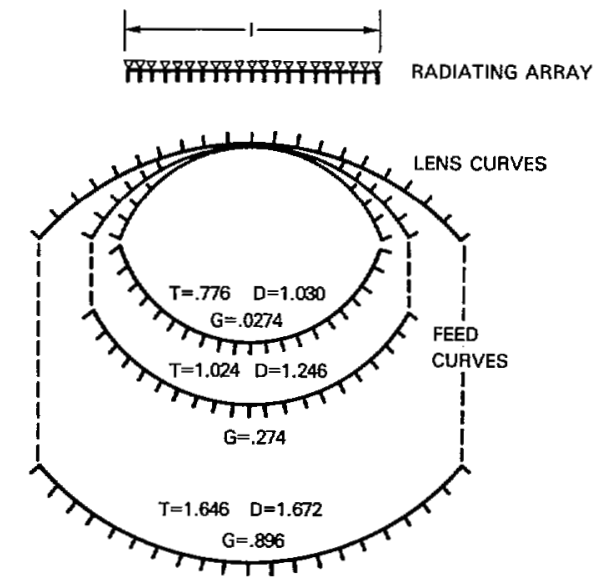

Fig. 7. Examples of symmetric Gent lenses.

ration by a factor of ten. If the allowable wavefront deviation is $\pm \lambda / 16$, it is found that the most compact lens-with $T=$ 0.78 -is usable with a maximum aperture size of $20 \lambda$. A lens of intermediate compactness, with $T=1$, is usable with an aperture as large as $60 \lambda$. For $T=1.5$, the aperture can be $180 \lambda$.

Many optical systems have aberration which is a power of some parameter such as the F/D ratio. The availability of such a relationship simplifies the analysis of the optical system in practical applications. An attempt was made to deduce a simple functional relationship between wavefront deviation and one of the lens parameters, but regrettably without success.

The element spacings on the feed and lens curves are consistent with phased array theory applied to arrays with limited angular coverage [7].

An efficient linear phased array radiator with ideally suited directive characteristics and coverage limits of $\theta_{1}$ and $\theta_{2}$ will 
have elements spaced according to

$$
s=\frac{\lambda}{\sin \theta_{2}-\sin \theta_{1}} .
$$

For the most compact lens with $G=0, T=3 / 4$, and $D=1$, the center elements have $\theta_{2}=\tan ^{-1}(4 / 3)$ and $\theta_{1}=-\tan ^{-1}(4 / 3)$, giving $s_{\min }=0.625 \lambda$. The edge elements have $\theta_{2}=\tan ^{-1} 3$ and $\theta_{1}=-\tan ^{-1}(1 / 3)$, giving $s_{\max }=0.7906 \lambda$. These values are seen to be close to the limiting values as $T \rightarrow 0.75$, of the $s_{\min }$ and $s_{\max }$ curves in Fig. 6 .

In order to obtain specific design information to be used in constructing a lens, one must use the equations presented in the Appendix. Fig. 8 of the Appendix should be examined at this point and the dimensional parameters defined thereon understood. Fig. 6 is useful in predicting the general dimensions and performance of a particular lens. The procedure is to select a candidate lens from Fig. 6, determine the appropriate value of the parameter $A$, and then enter the design equations at (A-7).

\section{GENERAL DISCUSSION OF BOOTLACE LENSES}

Although the primary purpose of this paper is to present theoretical design data for bootlace lenses, it is also worthwhile to examine the more general comparison between the characteristics of these lenses and "ideal" bootlace lenses. The ideal lens has characteristics which we would wish to obtain, while the actual lens is the type considered in this paper. A listing of the actual and ideal characteristics is presented in Table I.

Table I indicates that the conventional bootlace lens, although it is quite good, is far from ideal. It can be shown that items 1 and 3 are directly related. That is, if a lens can be devised with all line lengths equal, it will automatically require symmetric port radiation patterns. It can also be shown that if item 4 is satisfied, the lens can radiate directly without using the lens or radiator arrays or the interconnecting lines. That is, if item 4 is satisfied, the lens is no longer of the bootlace type. Thus for bootlace lenses, Table I can be reduced to three design features for comparing actual and ideal lenses-interconnecting line lengths, feed and lens curves, and amplitude distribution. J. P. Wild has proposed an ingenious bootlace lens configuration which satisfies the ideal requirements for these design features [8]. This lens differs from those discussed in this paper in that the parallel plate region is not planar.

No mention has been made of the focal length of these lenses, nor of other optical parameters such as field of view. Rotman and Turner used such terms, defining focal length as the center-line length of the wave transmission region and equating the angle to the off-axis focus to the coverage by requiring that the port spacing of the radiating array be equal to that at the center of the lens curve.

The design procedure of this paper emphasizes internal consistency in terms of array theory. The resultant lens-antenna system has a half-wave spaced array of $N$ radiators fed by an $N$-port lens. The $N$ beams will just cover the \pm 90 deg of visible space. Note that lenses defined in this way have performance equivalent to that of a Butler matrix. If we desire to obtain $M$ beams from an $N$-element array, where $M<N$, we do not utilize a smaller portion of the feed curve of an $N$-beam lens. Instead, we elect to fill the lens curve of an $M$-beam lens with $N$ elements. Therefore, all lens configurations are symmetric,

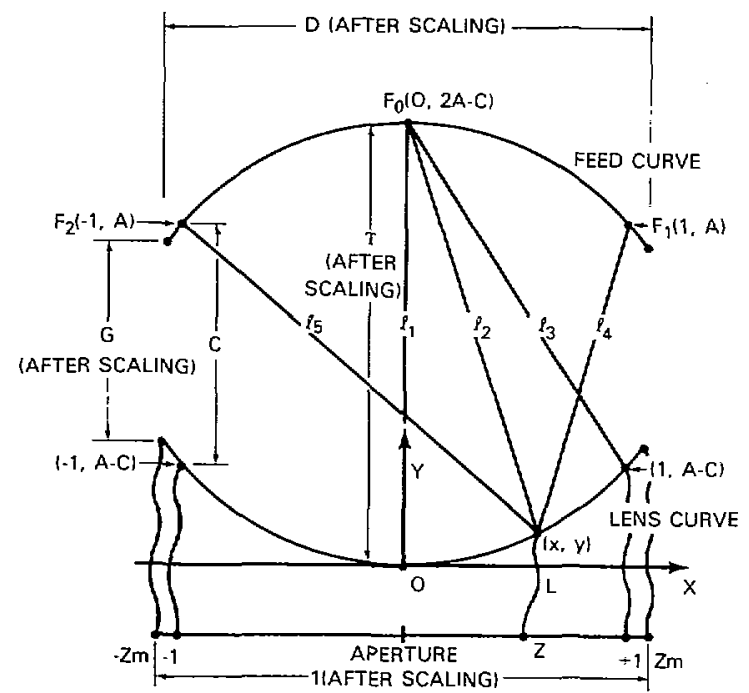

Fig. 8. Lens geometry used for design equations.

TABLE I

COMPARISON OF ACTUAL AND IDEAL LENS CHARACTERISTICS

\begin{tabular}{|c|c|c|}
\hline Design Feature & Actual Lens & Iäeal Lens \\
\hline $\begin{array}{l}\text { 1. Interconnecting trans- } \\
\text { mission lines }\end{array}$ & $\begin{array}{l}\text { Lines have variable } \\
\text { lengths }\end{array}$ & $\begin{array}{l}\text { All lines should } \\
\text { have equal length }\end{array}$ \\
\hline 2. Feed $\varepsilon$ lens curves & $\begin{array}{l}\text { Both curves have } \\
\text { strong curvature }\end{array}$ & $\begin{array}{l}\text { Both curves should } \\
\text { be straight Iines }\end{array}$ \\
\hline $\begin{array}{l}\text { 3. Recuired port radia- } \\
\text { tion patterns }\end{array}$ & $\begin{array}{l}\text { Patterns become } \\
\text { asymetric for } \\
\text { off-axis ports }\end{array}$ & $\begin{array}{l}\text { Patterns should be } \\
\text { symatric for all } \\
\text { ports }\end{array}$ \\
\hline 4. Port spacing function & $\begin{array}{l}\text { Port-spacing in- } \\
\text { creases for off- } \\
\text { axis ports }\end{array}$ & $\begin{array}{l}\text { Port spacing should } \\
\text { be constant for all } \\
\text { ports }\end{array}$ \\
\hline $\begin{array}{l}\text { 5. Oufput anplitude } \\
\text { cistribution }\end{array}$ & $\begin{array}{l}\text { Becones asymetric } \\
\text { for off-axis ports }\end{array}$ & $\begin{array}{l}\text { Should be uniform } \\
\text { or at least sym- } \\
\text { metric for all ports }\end{array}$ \\
\hline
\end{tabular}

whether $N \times N$ (elements $\times$ beams) or $N \times M$. This design rule has the effect of greatly reducing the complexity of the design process.

It may be somewhat disturbing to realize that the $N \times M$ configuration may result in arbitrarily small port spacings for the lens curve. Although this may present some practical difficulty, it is theoretically plausible. The lens ports will be mutually coupled and mismatched for small port spacings. However, the feed ports will be matched and the lens will be theoretically lossless as seen from the feed ports. This is analogous to the extreme case of obtaining a single beam from an array using a power divider. The power divider is mismatched when viewed from a single array port. Furthermore, a multibeam antenna array should ideally have one radiator for each beam, even for small overall angular coverage [9]. In a practical realization, careful engineering design should always yield $M \geqslant N / 2$, or no more than two radiators per beam. Thus the minimum element spacing on the lens curve will be about $0.3 \lambda$ to $0.4 \lambda$.

\section{CONCLUSION}

The focusing performance of a symmetric class of line-source, multiple-beam bootlace lenses has been evaluated. Design data for the lenses have been presented in graphical form. Design equations for generating detailed lens dimensions have also 
been presented. All designs have been normalized to produce \pm 90 -degree coverage from a $\lambda / 2$ spaced array of radiators. It is found that the most compact lens, with width about equal to the length of the array and thickness of 0.75 times the length of the array, can feed an aperture of $20 \lambda$ or about 40 elements. Increasing the size of the lens relative to the radiating array sharply reduces the wavefront aberration; doubling the thickness of the lens reduces aberration by a factor of ten, making apertures of $200 \lambda$ feasible.

\section{APPENDIX \\ DERIVATION OF LENS DESIGN EQUATIONS}

The initial configuration is shown in Fig. 8. The lens curve, plotted in an $x-y$ plane, is specified by the points $(0,0)$ and $( \pm 1, A-C)$. The foci are at $(0,2 A-C)$ and $( \pm 1, A)$. These six points are chosen so that the lens exhibits the desired symmetry. The corresponding portion of the radiating aperture covers the interval $-1<z<1$. Path lengths from the foci to arbitrary lens curve locations $(x, y)$, thence through the transmission lines $L$ to the radiating aperture at $z$ are indicated. The following guarantee the collimation of wavefronts from the three foci:

$$
\begin{aligned}
L(x, y)=L & =l_{1}-l_{2} \\
& =2 A-C-l_{2} .
\end{aligned}
$$

Equation (A-1) collimates all rays from $F_{0}$ :

$$
\begin{aligned}
& l_{4}+L=l_{3}-k z / 2 \\
& l_{5}+L=l_{3}+k z / 2 .
\end{aligned}
$$

Equations (A-2) and (A-3) collimate all rays from $F_{1}$ and $F_{2}$. The constant $k$ represents, for the off-axis foci $F_{1}$ and $F_{2}$, the path length difference between the rays to the aperture points $z= \pm 1$, and $k$ is determined by the assumed initial conditions.

Expressions for the path lengths are as follows:

$$
\begin{aligned}
& l_{3}=\sqrt{A^{2}+1} \\
& l_{4}=\sqrt{(x-1)^{2}+(A-y)^{2}} \\
& l_{5}=\sqrt{(x+1)^{2}+(A-y)^{2}} \\
& l_{2}=\sqrt{x^{2}+(2 A-C-y)^{2}} .
\end{aligned}
$$

Equations (A-2) and (A-3) become

$$
\begin{aligned}
& \sqrt{(x+1)^{2}+(A-y)^{2}}-\sqrt{(x-1)^{2}+(A-y)^{2}} \\
& \quad=\left(\sqrt{C^{2}+4}-C\right) z \\
& \sqrt{(x+1)^{2}+(A-y)^{2}}+\sqrt{(x-1)^{2}+(A-y)^{2}} \\
& \quad=2\left(\sqrt{A^{2}+1}-2 A+C+\sqrt{x^{2}+(2 A-C-y)^{2}}\right)
\end{aligned}
$$

where $k=\sqrt{C^{2}+4}-C$ since $x=1, y=A-C, z=1$ is an initial constraint. This same constraint applied to Equation (A-5) yields

$$
4\left(\sqrt{A^{2}+1}-A\right)=\sqrt{C^{2}+4}-C .
$$

Equations (A-4), (A-5), and (A-6) define a one-parameter family of lenses. The independent parameter is either $A$ or $C$, and (A-6) gives one in terms of the other. Then (A-4) and (A-5) are solved for $x$ and $y$ for any given value of $z$.

Equation (A-6) can be expressed parametrically as

$$
2 \tan \frac{w}{2}=\tan \frac{u}{2}
$$

where

$$
\begin{aligned}
& A=\cot w \\
& C=2 \cot u .
\end{aligned}
$$

Explicit solutions are

$$
\begin{aligned}
& A=\cot \left\{2 \tan ^{-1}\left[\frac{1}{2} \tan \left(\frac{1}{2} \cot ^{-1} \frac{C}{2}\right)\right]\right\} \\
& C=2 \cot \left\{2 \tan ^{-1}\left[2 \tan \left(\frac{1}{2} \cot ^{-1} A\right)\right]\right\} .
\end{aligned}
$$

Equations (A-4) and (A-5) are solved as follows. The value of $x$ is found from

$$
a\left(\frac{2 x}{U}\right)^{2}+b\left(\frac{2 x}{U}\right)+c=0
$$

taking the positive value of the root where

$$
\begin{aligned}
& a=4-U^{2}-\frac{V^{2}}{(A-C)^{2}} \\
& b=\frac{4 W V}{(A-C)^{2}} \\
& c=U^{2}-4-\frac{4 W^{2}}{(A-C)^{2}} \\
& W=\frac{V^{2}-U^{2}}{8}+\frac{1-(A-C)^{2}}{2} \\
& U=z\left(\sqrt{4+C^{2}}-C\right) \\
& V=2\left(-2 A+C+\sqrt{A^{2}+1}\right) .
\end{aligned}
$$

The value of $L$ is given by

$$
L=2 A-C-\sqrt{x^{2}+(2 A-C-y)^{2}} .
$$

The next step in the design procedure is to determine the extent of the lens and feed curves that can be used to provide the best possible performance from the lens. As indicated in Fig. 4, a maximum wavefront deviation $\delta_{m}$ is found on the interfocal region of the feed curve. Then the maximum usable value of $z$ is found by trying values of $z>1$ until the wavefront deviation equals $\delta_{m}$. It was found that $z_{m}$ is a function of $A$, and Figure 9 is a plot of this relationship. If the wavefront error function were exactly cubic in terms of $z$, then $z_{m}$ would be $2 / \sqrt{3}$, or 1.1547 . For $A=10, z_{m}=1.1500$, which supports the hypothesis that $z_{m}=1.1547$ is an asymptote of the curve of Figure 9. Figure 10 is a plot of the quantities 


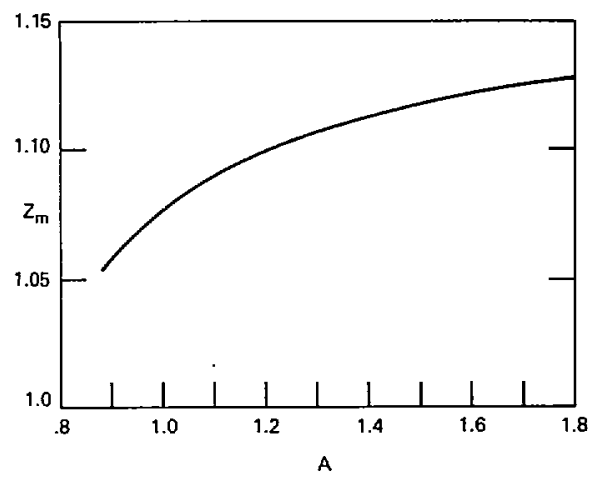

Fig. 9. $Z_{m}$ versus $A$.

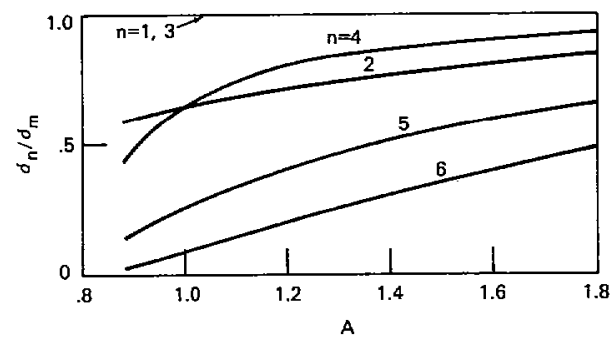

Fig. 10. $\delta_{n} / \delta_{m}$ versus $A$.

$\delta_{n} / \delta_{m}$ for $n=1-6$ as a function of $A$. Figure 10 shows clearly the variation of the error surface from the ideal cubic function for small $A$. The surface becomes more nearly cubic as $A$ increases. If Figure 10 were extended to $A=10$, it would be found that all values of $\delta_{n} / \delta_{m}$ exceed 0.86 .

Once the value of $z_{m}$ is determined for given $A$, it is possible to obtain lens dimensions for a radiating array of any number of elements by uniformly spacing the elements between $-z_{m}$ and $z_{m}$ and solving for $x, y$, and $L$.

The above design equations generated lenses that are "unscaled," so-called because the scale factor relating the lens size and the radiating array size was arbitrarily chosen. In order to best meet the needs of the design engineer, a scaling procedure is applied so that the edge feed position produces an end-fire beam from the array. Furthermore, the aperture length is given a value of unity, so that all dimensions are normalized in terms of the aperture. Methods for obtaining coverage other than \pm 90 degrees from the lens-array combination are discussed in Section IV. A scale factor of

$$
g=\frac{1}{\left[l_{3}\left(z_{m}\right)-l_{1}\left(z_{m}\right)\right] z_{m}}
$$

is multiplied by all values of $x, y, L$, and $\delta$ to describe the scaled lens dimensions. For the unscaled lens, the edge feed position, with $z=z_{m}$, produces a beam which makes an angle relative to the array normal given by $\sin \theta_{m}=\left[l_{3}\left(z_{m}\right)-\right.$ $\left.l_{1}\left(z_{m}\right)\right] / 2$, where $l_{3}\left(z_{m}\right)$ and $l_{1}\left(z_{m}\right)$ are path lengths from the edge of the feed curve to $x= \pm 1$ on the lens curve. In order to make $\theta_{m}=90$ deg we multiply the lens dimensions by $2 /\left[l_{3}\left(z_{m}\right)-l_{1}\left(z_{m}\right)\right]$. Then, in order to normalize all dimensions to an aperture of unity, we divide by $2 z_{m}$.

It is possible to obtain approximate relationships for the scaled lenses by assuming that $z_{m}=1$ and applying the scaling factor. It is then found that

$$
\begin{aligned}
& T=\frac{D^{2}}{2}+1 / 4 \\
& T=G+3 / 4,
\end{aligned}
$$

where $T$ is the lens thickness or spacing between center points of the lens and feed curves, $D$ is the lens width, and $G$ is the edge gap or minimum spacing between lens and feed curves. The smallest lens is obtained for $G=0, T=3 / 4$, and $D=1$.

\section{REFERENCES}

[1] H. Gent, "The bootlace aerial," Royal Radar Establishment J., pp. 47-57, Oct. 1957.

[2] S. S. D. Jones, H. Gent, and A. A. L. Browne, "Improvements in or relating to electromagnetic-wave lens and mirror systems," British Provisional Patent Specification No. 25926/56, Aug. 1956.

[3] M. L. Kales and R. M. Brown, "Two-dimensional symmetric bootlace lenses," IEEE Trans. Antennas Propagat., vol. AP-13, pp. 521-528, July 1965 .

[4] W. Rotman and R. F. Turner, "Wide-angle microwave lens for line source applications," IEEE Trans. Antennas Propagat., vol. AP-11, no. 6, pp. 623-632, Nov. 1963.

[5] D. Archer, "Lens-fed multiple-beam arrays," Microwave J., vol. 18, no. 10, pp. 37-42, Oct. 1975.

[6] R. M. Cox and J. R. Sebring, "MLS-A practical application of microwave technology," IEEE Trans. Microwave Theory Tech., vol. MTT-24, no. 12, pp. 964-971, Dec. 1976.

[7] W. K. Kahn, "Ideal efficiency of a radiating element in an infinite array," IEEE Trans. Antennas Propagat., vol. AP-15, pp. 534538 , July 1967.

[8] J. P. Wild, Radiophysics Lab., CSIRO, Sydney, Australia, private communication.

[9] R. S. Mailloux, "An overlapped subarray for limited scan application," IEEE Trans. Antennas Propagat., vol. AP-22, no. 3, pp. 487489, May 1974. 